\title{
ОСОБЕННОСТИ РАЗРАБОТКИ HDL-ПРОЕКTOB
}

ДЛЯ РЕАЛИЗАЦИИ

\section{В БАЗИСЕ ПЛИС СЕРИИ 5578ТС}

\section{П.Городков ${ }^{1}$}

УДК 621.382

BAK 05.27.00

По архитектурным решениям ПлИС серии 5578ТС, разработанные в АО "КТЦ "ЭЛЕКТРОНИКА", занимают промежуточное положение между низкобюджетной серией Altera Cyclone II и высокопроизводительными ПЛИС серии Altera Stratix III. Они построены на основе адаптивных логических модулей (АЛМ), что позволяет более эффективно расходовать ресурсы. Для разработчиков проектов на базе ПлИС практическое значение имеет анализ использования логических ресурсов. В статье рассмотрены особенности потребления логических ресурсов ПлИС данной серии при реализации проектов различной сложности с использованием типовых тестов производительности из набора Altera QUIP toolkit 9.0.

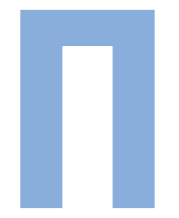

лИС серии 5578ТС представляют собой программируемые пользователем вентильные матрицы (ППВМ) с логическими блоками на основе адаптивных логических модулей (АЛМ). Логический блок ПлИС (рис.1) состоит из восьми АЛМ, каждый из которых имеет восемь входов данных и может реализовать одну произвольную 6-входовую или две произвольные 2-, 3-, 4-входовые функции [1]. АЛМ состоит из четырех 3-входовых и двух 4-входовых LUT, двух выделенных сумматоров и двух триггеров с возможностью синхронного и асинхронного сброса, синхронной загрузки из нескольких источников, использования обратной связи и обвода триггера (рис.2).

Воронежский государственный технический университет, аспирант кафедры полупроводниковой электроники и наноэлектроники, тел. (4732) 437695, gorodkoff@gmail.com.
Разработанный АЛМ по структуре схож с АЛМ плиС серии Stratix III [2], обеспечивающий повышенное быстродействие Плис и эффективность упаковки проектов пользователя благодаря расширенным режимам работы LUT5, LUT6 и встроенному сумматору для логико-арифметических вычислений. Однако на данный момент сумматор исключен из конфигурации, поскольку серия 5578ТС предназначена для замены ПЛИС ЕР2С5/ЕР2С8 от Altera.

При анализе использования логических ресурсов в ПлИС серии 5578ТС будем рассматривать следующие функциональные блоки:

- "LUT с триггерами" (реализация комбинационной и последовательностной логики);

- "только LUT";

- "только триггеры".

Кроме того, разработчик ПЛИС (АО "КТЦ "ЭЛЕКТРОНИКА") для анализа распределения ресурсов рекомен- 


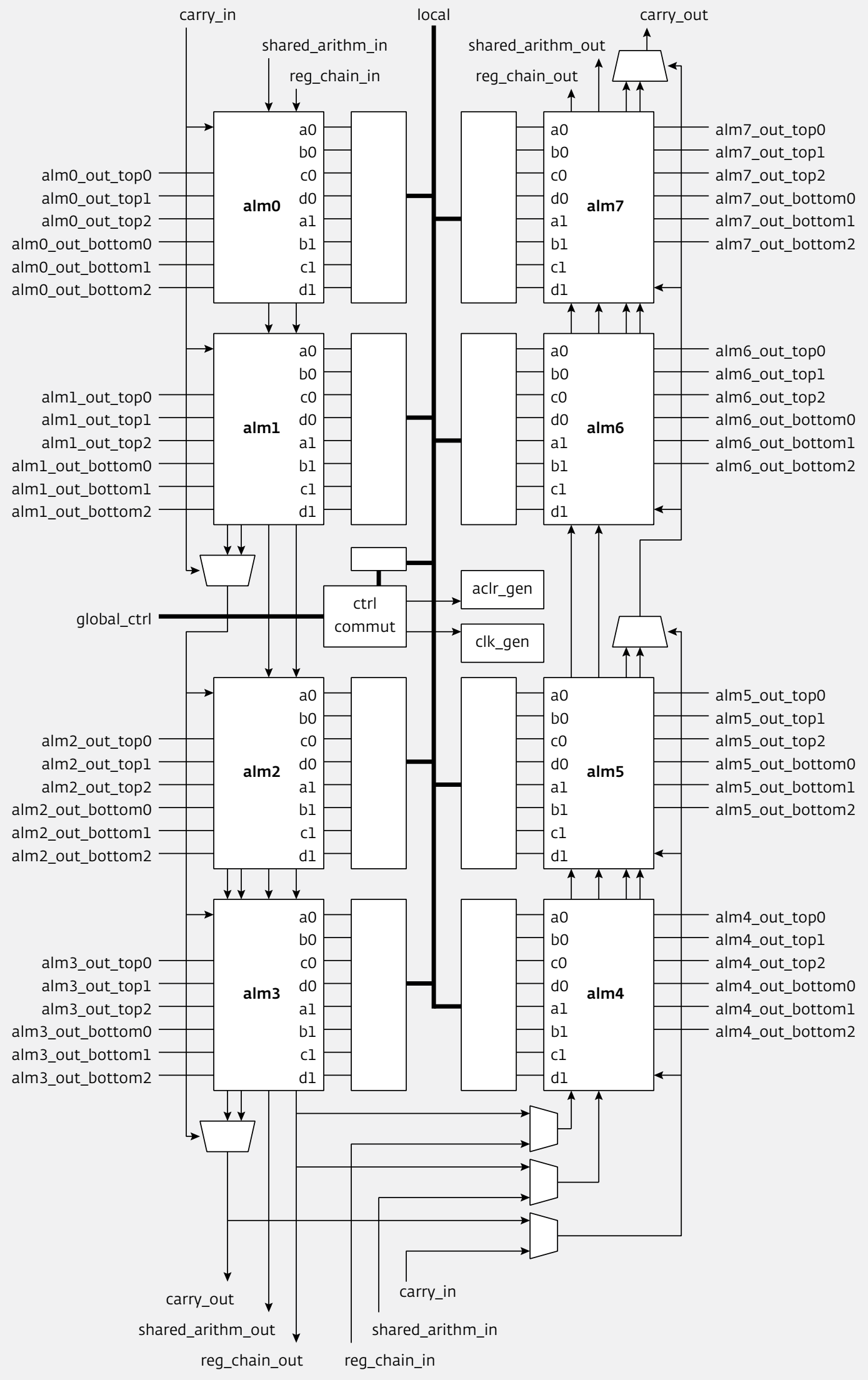

Рис.1. Схема логического блока Плис серии 5578TC 
дует использовать следующие параметры:

- alm_ff - число задействованных функциональных блоков "LUT с триггерами" и "только триггеры" для оценки использования последовательностной логики;

- Icell_comb - число задействованных функциональных блоков "LUT с триггерами" и "только LUT" для оценки использования комбинационной логики.

Для реализации решения в базисе отечественных ПлИС необходимо создать проект в среде проектирования Quartus версий 9.0-13.1 для ЕР2С5 в случае разработки для ПлИС 5578TC014 и ЕР2С8 для 5578ТС024.

Затем с помощью команды Analysis \& Synthesis выполнить анализ и синтез проекта и с помощью команды Start VQM Writer получить на выходе файл Verilog Quartus Mapping (VQM), представляющий собой неоптимизированный список логических соединений (netlist). При разработке проекта в Quartus важно следить за ограничениями, которые налагаются на используемые логические ресурсы (табл.1). Однако некоторые проекты, выходящие за пределы ограничений, могут успешно работать в базисе ПлИС серии 5578ТС, что можно установить лишь опытным путем.

Список соединений (netlist) загружается в среду разработки конфигурационных данных (СРКД), созданную в АО "КТЦ "ЭЛЕКТРОНИКА", которая производит анализ, размещение и трассировку ПлИС в соответствии с извлеченной информацией о логическом содержании проекта. При настройке проекта в СРКД можно выбрать режим работы АЛм с максимальной разрядностью LUT от 4 до 6 [2]. В последних обновлениях СРКД была добавлена возможность дополнительной оптими- зации проекта, которую в рамках данной статьи анализировать не будем, поскольку она зачастую увеличивает количество потребляемых ресурсов в целях повышения предельной тактовой частоты.

Распределение логических ресурсов в базисе Плис 5578ТС024 проанализируем с помощью типовых тестов производительности Плис (benchmark) из свободно распространяемого комплекта QUIP toolkit 9.0 на основе 26 проектов различной сложности из этого комплекта [3]. Размещение и трассировка проектов были выполнены в двух режимах работы АЛM: LUT4 и LUTG (табл.2-4).

Архитектуры ПлИС Cyclone II [4] и 5578TC различаются, поэтому проанализируем распределение LUT. Для наглядности полученное распределение для разных проектов представим в виде диаграммы (рис.3). Из нее видно, что в режиме 4-входовых LUT ПлИС 5578TC024 не выигрывает в потреблении логических ресурсов, тогда как режим 6-входовых LUT демонстрирует преимущества АЛм для большей части тестовых проектов.

Таблица 1. Ограничения логических ресурсов проекта Quartus II для ПлиС серии 5578TC

\begin{tabular}{|l|c|c|}
\hline \multirow{2}{*}{ Параметр } & \multicolumn{2}{|c|}{ Ограничение } \\
\cline { 2 - 3 } & $5578 \mathrm{TC} 014$ & 5578 TС024 \\
\hline Логические элементы в Quartus II & 3200 & 3400 \\
\hline alm_ff & 4032 & 5760 \\
\hline lcell_comb & 4032 & 14 \\
\hline Умножители (18×18) & 252 Кбит \\
\hline Память & 180 \\
\hline Выводы (включая восемь глобальных тактовых входов) & 360 Кбит \\
\hline
\end{tabular}


Таблица 2. Распределение логических ресурсов ПлиС серии 5578TC в режиме LUT6

\begin{tabular}{|c|c|c|c|c|c|c|}
\hline № & Проект & $\begin{array}{c}\text { Триггеры и триг- } \\
\text { геры c LUT/ LUT } \\
\text { (alm_ff/lcell_comb) }\end{array}$ & $\begin{array}{c}\text { LUT } \\
\text { с триг- } \\
\text { герами }\end{array}$ & $\begin{array}{l}\text { Только } \\
\text { LUT }\end{array}$ & $\begin{array}{l}\text { Только } \\
\text { триг- } \\
\text { геры }\end{array}$ & Описание, комментарии \\
\hline \multicolumn{7}{|c|}{ Простые HDL-проекты } \\
\hline 1 & Barrel16 & $37 / 88$ & 36 & 52 & 1 & \multirow{4}{*}{$\begin{array}{l}\text { Устройства быстрого сдвига } \\
\text { (barrel shifter) разной разряд- } \\
\text { ности }\end{array}$} \\
\hline 2 & Barrel16a & $37 / 126$ & 21 & 105 & 16 & \\
\hline 3 & Barrel32 & $70 / 244$ & 66 & 178 & 4 & \\
\hline 4 & Barrel64 & $135 / 488$ & 112 & 376 & 23 & \\
\hline 5 & Mux32_16bit & $533 / 176$ & 136 & 40 & 397 & \multirow{4}{*}{$\begin{array}{l}\text { Шины мультиплексоров разной } \\
\text { разрядности }\end{array}$} \\
\hline 6 & Mux64_16bit & $1046 / 352$ & 265 & 87 & 781 & \\
\hline 7 & Mux8_128bit & $1155 / 392$ & 375 & 17 & 780 & \\
\hline 8 & Mux8_64bit & $579 / 200$ & 185 & 15 & 394 & \\
\hline 9 & Xbar_16x16 & $32 / 80$ & 16 & 64 & 16 & Коммутатор (16 бит) \\
\hline 10 & Ts_mike_fsm & $3 / 11$ & 3 & 8 & 0 & Конечный автомат \\
\hline 11 & Fip_cordic_cla & $55 / 304$ & 55 & 249 & 0 & \multirow{2}{*}{$\begin{array}{l}\text { Вычислитель поворота системы } \\
\text { координат }\end{array}$} \\
\hline 12 & Fip_cordic_rca & $55 / 335$ & 55 & 280 & 0 & \\
\hline 13 & oc_fcmp & $0 / 157$ & 0 & 157 & 0 & $\begin{array}{l}\text { Компаратор одинарной точ- } \\
\text { ности для чисел с плавающей } \\
\text { точкой }\end{array}$ \\
\hline \multicolumn{7}{|c|}{ Интерфейсы } \\
\hline 14 & oc_miniuart & $73 / 111$ & 67 & 44 & 6 & Ядро miniUART \\
\hline 15 & oc_sdram & $112 / 188$ & 106 & 82 & 6 & Контроллер SDRAM \\
\hline 16 & os_sdram 16 & $147 / 275$ & 141 & 134 & 6 & Контроллер SDRAM \\
\hline 17 & oc_gpio & $100 / 118$ & 42 & 76 & 58 & Ядро GPIO IP \\
\hline 18 & oc_ata_ocidec1 & $269 / 271$ & 138 & 133 & 131 & $\begin{array}{l}\text { Контроллер IDE ATA / ATAPI-5 } \\
\text { (OCIDEC-1) }\end{array}$ \\
\hline \multicolumn{7}{|c|}{ ЦОС-алгоритмы } \\
\hline 19 & oc_correlator & $219 / 261$ & 126 & 135 & 93 & Коррелятор для Bluetooth \\
\hline 20 & oc_dct_slow & $178 / 235$ & 174 & 61 & 4 & $\begin{array}{l}\text { Дискретное косинусное преоб- } \\
\text { разование }\end{array}$ \\
\hline 21 & $\begin{array}{l}\text { oc_video_compression_ } \\
\text { systems_huffman_enc }\end{array}$ & $59 / 304$ & 58 & 246 & 1 & $\begin{array}{l}\text { Алгоритм сжатия изображения } \\
\text { Хаффмана }\end{array}$ \\
\hline 22 & $\begin{array}{l}\text { oc_video_compression_ } \\
\text { systems_huffman_dec }\end{array}$ & $62 / 672$ & 62 & 610 & 0 & $\begin{array}{l}\text { Алгоритм ЦОС для декодиро- } \\
\text { вания сжатых изображений } \\
\text { по алгоритму Хаффмана }\end{array}$ \\
\hline 23 & oc_simple_fm_receiver & $230 / 1241$ & 230 & 1011 & 0 & $\begin{array}{l}\text { Радиоприемник по протоколу } \\
\text { Bluetooth }\end{array}$ \\
\hline \multicolumn{7}{|c|}{ Прочие проекты } \\
\hline 24 & Pong & $157 / 648$ & 147 & 501 & 10 & Академическая игра PONG \\
\hline 25 & fip_risc8 & $660 / 924$ & 364 & 560 & 296 & Процессор архитектуры RISC \\
\hline 26 & oc_rtc & $114 / 342$ & 112 & 230 & 2 & $\begin{array}{l}\text { Ядро микроконтроллера реаль- } \\
\text { ного времени }\end{array}$ \\
\hline
\end{tabular}


Таблица 3. Распределение логических ресурсов ПлиС серии 5578TC в режиме LUT4

\begin{tabular}{|c|c|c|c|c|c|c|}
\hline № & Проект & $\begin{array}{l}\text { Триггеры и триг- } \\
\text { геры с LUT/ LUT } \\
\text { (alm_ff/lcell_comb) }\end{array}$ & $\begin{array}{l}\text { LUT } \\
\text { с триг- } \\
\text { герами }\end{array}$ & $\begin{array}{l}\text { Только } \\
\text { LUT }\end{array}$ & $\begin{array}{c}\text { Только } \\
\text { триг- } \\
\text { геры }\end{array}$ & Описание, комментарии \\
\hline \multicolumn{7}{|c|}{ Простые HDL-проекты } \\
\hline 1 & Barrel16 & $37 / 116$ & 37 & 79 & 0 & \multirow{4}{*}{$\begin{array}{l}\text { Устройства быстрого сдвига (barrel } \\
\text { shifter) разной разрядности }\end{array}$} \\
\hline 2 & Barrel16a & $37 / 131$ & 37 & 94 & 0 & \\
\hline 3 & Barrel32 & $70 / 422$ & 65 & 357 & 5 & \\
\hline 4 & Barrel64 & $135 / 1077$ & 135 & 942 & 0 & \\
\hline 5 & Mux32_16bit & $533 / 336$ & 292 & 44 & 241 & \multirow{4}{*}{$\begin{array}{l}\text { Шины мультиплексоров разной } \\
\text { разрядности }\end{array}$} \\
\hline 6 & Mux64_16bit & $1046 / 672$ & 578 & 94 & 468 & \\
\hline 7 & Mux8_128bit & $1155 / 640$ & 631 & 9 & 524 & \\
\hline 8 & Mux8_64bit & $579 / 320$ & 303 & 17 & 276 & \\
\hline 9 & Xbar_16x16 & $32 / 160$ & 32 & 128 & 0 & Коммутатор (16 бит) \\
\hline 10 & Ts_mike_fsm & $3 / 18$ & 3 & 15 & 0 & Конечный автомат \\
\hline 11 & Fip_cordic_cla & $55 / 404$ & 55 & 349 & 0 & \multirow{2}{*}{$\begin{array}{l}\text { Вычислитель поворота системы } \\
\text { координат }\end{array}$} \\
\hline 12 & Fip_cordic_rca & $55 / 415$ & 55 & 360 & 0 & \\
\hline \multicolumn{7}{|c|}{ Интерфейсы } \\
\hline 13 & oc_fcmp & $0 / 132$ & 0 & 132 & 0 & $\begin{array}{l}\text { Компаратор одинарной точности } \\
\text { для чисел с плавающей точкой }\end{array}$ \\
\hline 14 & oc_miniuart & $73 / 155$ & 73 & 82 & 0 & Ядро miniUART \\
\hline 15 & oc_sdram & $112 / 188$ & 106 & 82 & 6 & \multirow{2}{*}{ Контроллер SDRAM } \\
\hline 16 & os_sdram16 & $147 / 344$ & 146 & 198 & 1 & \\
\hline 17 & oc_gpio & $100 / 140$ & 80 & 60 & 20 & Ядро GPIO IP \\
\hline 18 & oc_ata_ocidec1 & $269 / 367$ & 247 & 120 & 22 & $\begin{array}{l}\text { Контроллер IDE ATA / ATAPI-5 } \\
\text { (OCIDEC-1) }\end{array}$ \\
\hline \multicolumn{7}{|c|}{ ЦОС-алгоритмы } \\
\hline 19 & oc_correlator & $219 / 261$ & 135 & 126 & 84 & Коррелятор для Bluetooth \\
\hline 20 & oc_dct_slow & $178 / 299$ & 178 & 121 & 0 & $\begin{array}{l}\text { Дискретное косинусное преобра- } \\
\text { зование }\end{array}$ \\
\hline 21 & $\begin{array}{l}\text { oc_video_compression_ } \\
\text { systems_huffman_enc }\end{array}$ & $59 / 655$ & 59 & 596 & 0 & $\begin{array}{l}\text { Алгоритм сжатия изображения } \\
\text { Хаффмана }\end{array}$ \\
\hline 22 & $\begin{array}{l}\text { oc_video_compression_- } \\
\text { systems_huffman_dec }\end{array}$ & $62 / 477$ & 51 & 426 & 11 & $\begin{array}{l}\text { Алгоритм ЦОС для декодирования } \\
\text { сжатых изображений по алго- } \\
\text { ритму Хаффмана }\end{array}$ \\
\hline 23 & oc_simple_fm_receiver & $230 / 633$ & 194 & 439 & 36 & $\begin{array}{l}\text { Радиоприемник по протоколу } \\
\text { Bluetooth }\end{array}$ \\
\hline \multicolumn{7}{|c|}{ Прочие проекты } \\
\hline 24 & Pong & $157 / 696$ & 157 & 539 & 0 & Академическая игра PONG \\
\hline 25 & fip_risc8 & $660 / 644$ & 146 & 498 & 514 & Процессор архитектуры RISC \\
\hline 26 & oc_rtc & $114 / 247$ & 99 & 148 & 15 & $\begin{array}{l}\text { Ядро микроконтроллера реального } \\
\text { времени }\end{array}$ \\
\hline
\end{tabular}


Таблица 4. Распределение логических ресурсов Плис ЕР2С8

\begin{tabular}{|c|c|c|c|c|c|}
\hline № & Проект & $\begin{array}{l}\text { Логические } \\
\text { элементы }\end{array}$ & LUT & Триггеры & Описание, комментарии \\
\hline \multicolumn{6}{|c|}{ Простые HDL-проекты } \\
\hline 1 & Barrel16 & 118 & 116 & 37 & \multirow{4}{*}{$\begin{array}{l}\text { Устройства быстрого сдвига (barrel } \\
\text { shifter) разной разрядности }\end{array}$} \\
\hline 2 & Barrel16a & 132 & 131 & 37 & \\
\hline 3 & Barrel32 & 299 & 296 & 70 & \\
\hline 4 & Barrel64 & 988 & 986 & 135 & \\
\hline 5 & Mux32_16bit & 611 & 336 & 533 & \multirow{4}{*}{$\begin{array}{l}\text { Шины мультиплексоров разной раз- } \\
\text { рядности }\end{array}$} \\
\hline 6 & Mux64_16bit & 1,228 & 672 & 1046 & \\
\hline 7 & Mux8_128bit & 1,162 & 640 & 1155 & \\
\hline 8 & Mux8_64bit & 581 & 320 & 579 & \\
\hline 9 & Xbar_16x16 & 160 & 160 & 32 & Коммутатор (16 бит) \\
\hline 10 & Ts_mike_fsm & 14 & 14 & 3 & Простой конечный автомат \\
\hline 11 & Fip_cordic_cla & 411 & 403 & 55 & \multirow{2}{*}{$\begin{array}{l}\text { Вычислитель поворота системы } \\
\text { координат }\end{array}$} \\
\hline 12 & Fip_cordic_rca & 422 & 414 & 55 & \\
\hline \multicolumn{6}{|c|}{ Интерфейсы } \\
\hline 13 & oc_fcmp & 156 & 156 & 0 & $\begin{array}{l}\text { Компаратор одинарной точности } \\
\text { для чисел с плавающей точкой }\end{array}$ \\
\hline 14 & oc_miniuart & 156 & 73 & 154 & Ядро miniUART \\
\hline 15 & oc_sdram & 234 & 234 & 112 & \multirow{2}{*}{ Контроллер SDRAM } \\
\hline 16 & os_sdram16 & 302 & 297 & 147 & \\
\hline 17 & oc_gpio & 160 & 124 & 100 & Ядро GPIO IP \\
\hline 18 & oc_ata_ocidec1 & 395 & 331 & 269 & $\begin{array}{l}\text { Контроллер IDE ATA / ATAPI-5 } \\
\text { (OCIDEC-1) }\end{array}$ \\
\hline \multicolumn{6}{|c|}{ ЦОС-алгоритмы } \\
\hline 19 & oc_correlator & 346 & 241 & 219 & Коррелятор для Bluetooth \\
\hline 20 & oc_dct_slow & 284 & 283 & 178 & $\begin{array}{l}\text { Дискретное косинусное преобразо- } \\
\text { вание }\end{array}$ \\
\hline 21 & $\begin{array}{l}\text { oc_video_compression_ } \\
\text { systems_huffman_enc }\end{array}$ & 599 & 597 & 59 & $\begin{array}{l}\text { Алгоритм сжатия изображения } \\
\text { Хаффмана }\end{array}$ \\
\hline 22 & $\begin{array}{l}\text { oc_video_compression_ } \\
\text { systems_huffman_dec }\end{array}$ & 652 & 647 & 62 & $\begin{array}{l}\text { Алгоритм ЦОС для декодирования } \\
\text { сжатых изображений по алгоритму } \\
\text { Хаффмана }\end{array}$ \\
\hline 23 & oc_simple_fm_receiver & 1146 & 1114 & 230 & $\begin{array}{l}\text { Радиоприемник по протоколу } \\
\text { Bluetooth }\end{array}$ \\
\hline \multicolumn{6}{|c|}{ Прочие проекты } \\
\hline 24 & Pong & 679 & 652 & 157 & Академическая игра PONG \\
\hline 25 & fip_risc8 & 1158 & 897 & 660 & Процессор архитектуры RISC \\
\hline 26 & oc_rtc & 337 & 334 & 114 & $\begin{array}{l}\text { Ядро микроконтроллера реального } \\
\text { времени }\end{array}$ \\
\hline
\end{tabular}


Таблица 5. Нереализуемые в базисе ПлИС 5578TC024 проекты QUIP toolkit

\begin{tabular}{|c|c|c|c|}
\hline Проект & $\begin{array}{c}\text { Логические } \\
\text { элементы } \\
\text { ЕР2С8 }\end{array}$ & Ошибка & Описание, комментарии \\
\hline oc_oc8051 & 2708 & \multirow{3}{*}{$\begin{array}{l}\text { Используется режим работы } \\
\text { памяти с одновременным чтением } \\
\text { и записью по одному адресу }\end{array}$} & Ядро микроконтроллера 8051 (MCS-51 Intel) \\
\hline oc_minirisc & 475 & & $\begin{array}{l}\text { Ядро микроконтроллера MiniRisc } \\
\text { (совместим с PIC16С57 от Microchip) }\end{array}$ \\
\hline oc_hdlc & 561 & & Контроллер High-Level Data Link Control \\
\hline oc_pavr & 3847 & $\begin{array}{l}\text { Превышение используемых логи- } \\
\text { ческих ресурсов на 53\% }\end{array}$ & $\begin{array}{l}\text { Конвейерный 8-битный RICS-контроллер } \\
\text { (совместим с ядром Atmel AVR (6 стадий } \\
\text { конвейерной обработки) }\end{array}$ \\
\hline oc_fpu & 6724 & $\begin{array}{l}\text { Превышение используемых логи- } \\
\text { ческих ресурсов на 19\% }\end{array}$ & $\begin{array}{l}\text { Математические операции для чисел } \\
\text { с плавающей точкой }\end{array}$ \\
\hline oc_aquarius & 5048 & $\begin{array}{l}\text { Превышение используемых логи- } \\
\text { ческих ресурсов на 16\% }\end{array}$ & $\begin{array}{l}\text { Конвейерный RISC-микроконтроллер, } \\
\text { может исполнять инструкции формата } \\
\text { SuperH-2 }\end{array}$ \\
\hline
\end{tabular}

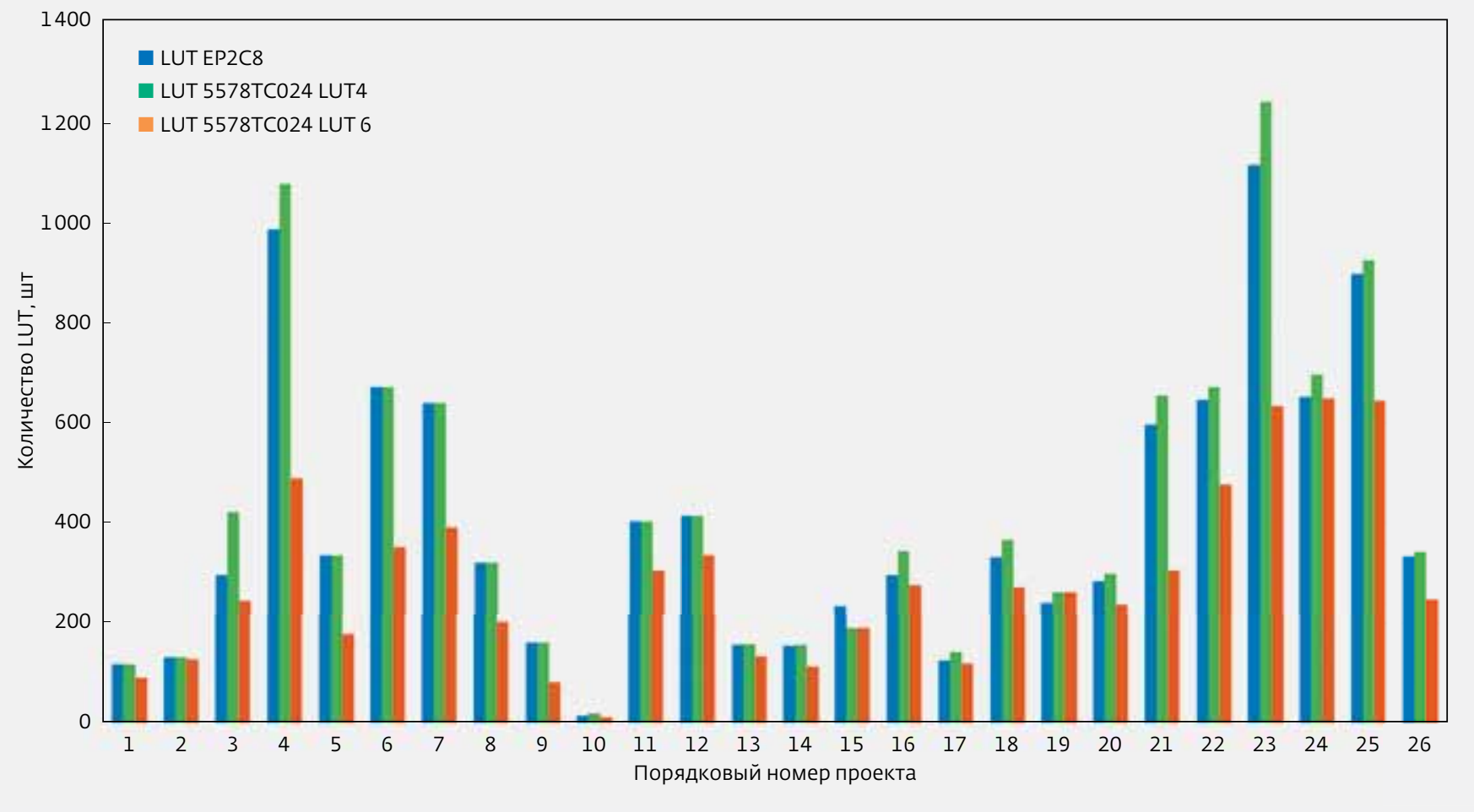

Рис.3. Распределение LUT в проектах (табл.2-4) для ПЛИС 5578TC024 и ЕР2C8 
В 22-х из 26-ти тестовых проектов потребление логических ресурсов снизилось в среднем на $25 \%$.

Часть проектов из набора QUIP toolkit, которые успешно раскладываются в базис ПлИС ЕР2С8, не удалось реализовать в базис ПлиС серии 5578ТС. Блоки памяти ПЛИС серии 5578ТС не поддерживают режим одновременного чтения и записи по одному адресу (в Quartus II это опция мегафункции RAM: 2-PORT Old memory contents appear). Поддерживается только режим I do not care, в котором значение выхода в момент записи будет неопределенным. Следует учитывать, что данное ограничение повлияет на работоспособность всех мегафункций, которые явно или неявно используют данный режим работы памяти, например таких, как ALTACCUMULATE, ALT_MULT_ACCUM, ALT_MEM_MULT. В табл. 5 указаны все проекты, реализованные в базисе ЕР2С8, но завершившиеся с ошибкой для ПлИС серии 5578TC.

$$
\text { \% }
$$

В заключение следует отметить, что плИС 5578ТС014 и 5578ТС024 не являются прямыми аналогами ПлИС серий Altera Cyclone II EP2C5 и EP2C8, а предназначены для их замены. В ПлИС серии 5578ТС используются АЛМ, близкие по своей архитектуре к семейству Altera Stratix III $[5,6]$.
Полученные результаты представляют практическую ценность для разработчиков проектов в базисе ПлИС серии 5578ТС, поскольку в настоящее время нет публикаций с анализом потребления логических ресурсов ПлИС данной серии.

\section{ЛИТЕРАТУРА}

1. Каталог изделий АО "ВЗПП-C".- www.vzpp-s.ru/ production/catalog.pdf.

2. Строгонов А.В., Городков П.С. Особенности проектирования устройств цифровой обработки сигналов в базисе ПлиС серии 5578 // Вестник ВГТУ. 2016. Т. 12. № 3. C. 51-56.

3. Altera. Benchmark Designs For The Quartus University Interface Program (QUIP).- https://github.com/neilisaac/ ece496/blob/master/reference/quip_toolkit-9.0/ documents/quip_benchmarks.pdf.

4. Altera Corporation Cyclone II Device Handbook, Vol. 1, February 2007, ver. 3.3.-www.altera.com/literature/hb/ cyc2/cyc2_cii5v1.pdf.

5. Altera Corporation Stratix III Device Handbook, Vol. 1, July 2010, ver. 2.3.- www.altera.com/literature/hb/stx3/stx3_ siii5v1.pdf.

6. Золотухо Р., Комолов Д. Stratix III-новое семейство FPGA фирмы Altera // Компоненты и технологии. 2006. № 12 . 